Fabrication of carbon nanotube nanogap electrodes by helium ion sputtering for molecular contacts

Cornelius Thiele', Henning Vieker, André Beyer, Benjamin S. Flavel, Frank Hennrich, David Muñoz Torres, Thomas R. Eaton, Marcel Mayor, Manfred M. Kappes, Armin Gölzhäuser, Hilbert v. Löhneysen, and Ralph Krupke'

Citation: Appl. Phys. Lett. 104, 103102 (2014); doi: 10.1063/1.4868097

View online: http://dx.doi.org/10.1063/1.4868097

View Table of Contents: http://aip.scitation.org/toc/apl/104/10

Published by the American Institute of Physics
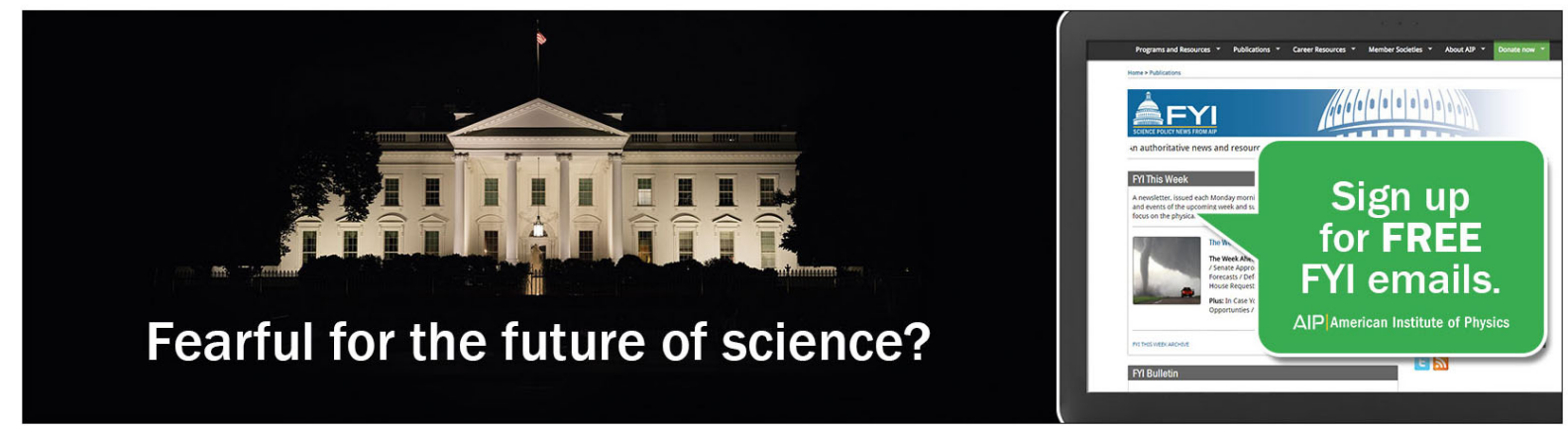


\title{
Fabrication of carbon nanotube nanogap electrodes by helium ion sputtering for molecular contacts
}

\author{
Cornelius Thiele, ${ }^{1,2, a)}$ Henning Vieker, ${ }^{3}$ André Beyer, ${ }^{3}$ Benjamin S. Flavel, ${ }^{1}$ \\ Frank Hennrich, ${ }^{1}$ David Muñoz Torres, ${ }^{4}$ Thomas R. Eaton, ${ }^{4}$ Marcel Mayor, ${ }^{1,2,4}$ \\ Manfred M. Kappes, ${ }^{1,2,5}$ Armin Gölzhäuser, ${ }^{3}$ Hilbert v. Löhneysen, ${ }^{1,2,6,7}$ \\ and Ralph Krupke $\mathrm{i}^{1,2,8, \mathrm{~b})}$ \\ ${ }^{1}$ Institute of Nanotechnology, Karlsruhe Institute of Technology, 76021 Karlsruhe, Germany \\ ${ }^{2}$ DFG Center for Functional Nanostructures (CFN), 76028 Karlsruhe, Germany \\ ${ }^{3}$ Faculty of Physics, Bielefeld University, 33615 Bielefeld, Germany \\ ${ }^{4}$ Department of Chemistry, University of Basel, 4056 Basel, Switzerland \\ ${ }^{5}$ Institut für Physikalische Chemie, Karlsruhe Institute of Technology, 76131 Karlsruhe, Germany \\ ${ }^{6}$ Physikalisches Institut, Karlsruhe Institute of Technology, 76128 Karlsruhe, Germany \\ ${ }^{7}$ Institut für Festkörperphysik, Karlsruhe Institute of Technology, 76021 Karlsruhe, Germany \\ ${ }^{8}$ Institut für Materialwissenschaft, Technische Universität Darmstadt, 64287 Darmstadt, Germany
}

(Received 6 February 2014; accepted 24 February 2014; published online 10 March 2014)

\begin{abstract}
Carbon nanotube nanogaps have been used to contact individual organic molecules. However, the reliable fabrication of a truly nanometer-sized gap remains a challenge. We use helium ion beam lithography to sputter nanogaps of only $(2.8 \pm 0.6) \mathrm{nm}$ size into single metallic carbon nanotubes embedded in a device geometry. The high reproducibility of the gap size formation provides a reliable nanogap electrode testbed for contacting small organic molecules. To demonstrate the functionality of these nanogap electrodes, we integrate oligo(phenylene ethynylene) molecular rods, and measure resistance before and after gap formation and with and without contacted molecules. (C) 2014 AIP Publishing LLC. [http://dx.doi.org/10.1063/1.4868097]
\end{abstract}

The use of individual metallic carbon nanotubes (CNTs) as electrodes is attractive for contacting nanocrystals, ${ }^{1}$ single molecules, ${ }^{2}$ and functional materials, such as phase-change materials, ${ }^{3}$ due to the CNT's intrinsic one-dimensionality and electrical conductivity. The formation of CNT electrodes starts, typically, with a pristine CNT on a surface, where the nanotube is contacted by lithographically defined metallic electrodes. Subsequently, two opposing CNT electrodes are generated by forming a gap in the CNT near its center. The molecule or material of interest is assembled between or deposited onto the CNT electrodes.

Different methods for fabricating such nanogaps have been reported. For example, current-induced breakdown in high vacuum, ${ }^{1}$ which has been shown to produce gaps down to $7 \mathrm{~nm}$, however, the typical gap size is often much larger. Alternatively, plasma oxidation through a lithographic mask, a complicated technique, limited by reliability problems and highly variable gap sizes. ${ }^{3}$ The use of electron-beam-induced oxidation has been shown to overcome variability in gap size, ${ }^{4}$ but resulted in a typical gap of $\approx 20 \mathrm{~nm}$, which is an order of magnitude too large for most organic molecules.

In this work, we report on the use of a helium ion microscope (HIM) to reliably fabricate nanogaps of $(2.8 \pm 0.6)$ $\mathrm{nm}$ in metallic single-walled carbon nanotubes (mSWNTs), see Fig. 1(a) for a scheme. The optimum helium ion sputtering condition for gap formation was determined by in-situ voltage-contrast microscopy analysis. The functionality of the nanogaps was demonstrated by contacting of oligo(phenylene ethynylene) (OPE) molecular rods and electrical characterization throughout the fabrication process.

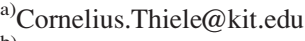

b)krupke@kit.edu
}

Tungsten electrodes with a separation of $700 \mathrm{~nm}$ were fabricated on a silicon substrate with $800 \mathrm{~nm}$ of thermal oxide, using a two-layer photoresist system comprising $180 \mathrm{~nm}$ poly(methyl methacrylate) (PMMA) 600 K EL11 and $200 \mathrm{~nm}$ of PMMA 950 K A4.5. Standard electron-beam lithography was used to pattern the photoresist. To obtain a nearly flat sample surface, metallic electrodes were "buried" into the oxide by etching their pattern into the surface with a $\mathrm{CHF}_{3}$ plasma. Sputter deposition was then used to fill these electrode trenches with tungsten. Finally, the photoresist and the undesired metal were lifted off in an acetone bath.

The mSWNTs were prepared by S-200 gel filtration and density-gradient ultracentrifugation (DGU). For an initial suspension, typically, $10 \mathrm{mg}$ of raw CNT material from pulsed laser vaporization ${ }^{5}$ was suspended in $15 \mathrm{ml} \mathrm{H}_{2} \mathrm{O}$ with $1 \mathrm{wt}$ \% of sodium dodecyl sulfate (SDS) using a tip sonicator (Bandelin, $200 \mathrm{~W}$ maximum power, $20 \mathrm{kHz}, 100 \mathrm{~ms}$ pulses) for $2 \mathrm{~h}$ at $\approx 20 \%$ power. During sonication, the suspension was cooled by a $500 \mathrm{ml}$ water bath. The resulting dispersion was then centrifuged with $\approx 100.000 \mathrm{~g}$ for $1.5 \mathrm{~h}$ and carefully decanted from the pellet, which was formed during centrifugation. The centrifuged CNT suspension was used as the starting suspension for gel filtration fractionation. Gel filtration was performed in a glass column of $20 \mathrm{~cm}$ length and $2 \mathrm{~cm}$ inner diameter. After filling the glass column with the filtration medium, the gel was slightly compressed to yield a final height of $\approx 14 \mathrm{~cm}$. For the separation, $\approx 10 \mathrm{ml}$ of initial suspension was applied to the top of the column and subsequently a solution of 1 wt. \% SDS in $\mathrm{H}_{2} \mathrm{O}$ as eluant was pushed through the column with compressed air by applying sufficient pressure to ensure a flow of $\approx 1 \mathrm{ml} / \mathrm{min}$. After $\approx 10 \mathrm{ml}$ of this eluant had been added most of the mSWNTs had moved through the column, whereas the semiconducting 
(a)

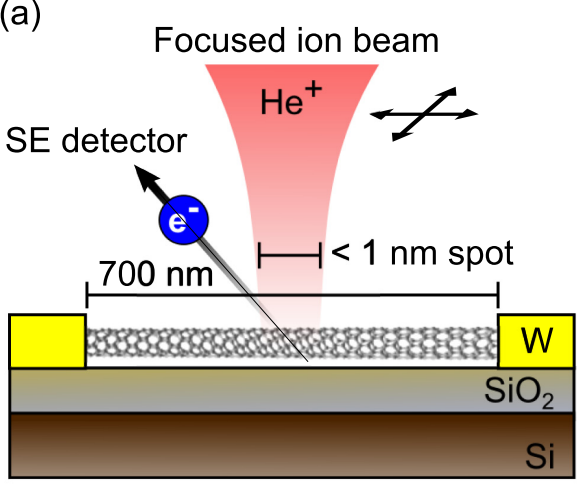

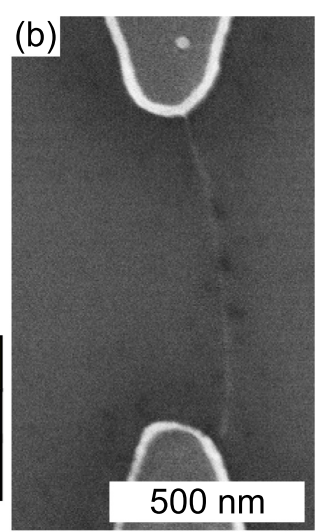
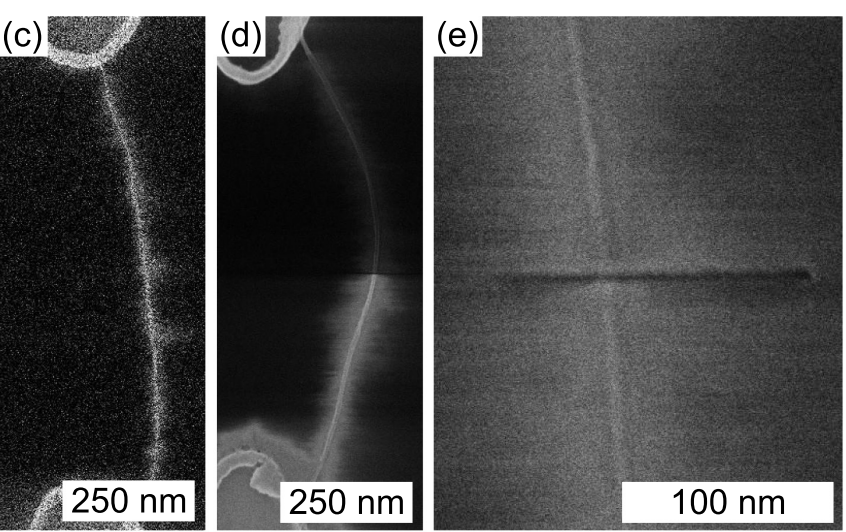

FIG. 1. (a) Scheme of helium ion sputtering of mSWNTs embedded in a device geometry. The nanotubes are deposited on tungsten electrodes and supported by $\mathrm{SiO}_{2} / \mathrm{Si}$ substrates. (b) SEM image of a mSWNT device after dielectrophoretic deposition. (c) Fast alignment scan of the same device with the helium ion microscope. (d) Slow scan of a device after gap formation. One of the electrodes is now electrically floating and accumulates positive charges during scanning. (e) Slow scan of a device with both electrodes grounded after gap formation. A clear trench is visible, also in the silicon oxide substrate.

CNTs remained trapped in the upper part of the gel. ${ }^{6}$ The mSWNTs were collected and used for DGU, to remove defected mSWNTs and any additional carbonaceous species present. Ultracentrifugation was performed in $20 \mathrm{wt} . \%$ iodixanol and $1 \mathrm{wt}$. \% SDS in $\mathrm{H}_{2} \mathrm{O}$. mSWNTs with a diameter distribution of $(1.2 \pm 0.2) \mathrm{nm}$ were used in this work.

mSWNTs were deposited between the tungsten electrodes using dielectrophoresis. ${ }^{7}$ An alternating voltage with a frequency of $300 \mathrm{kHz}$ and a peak-to-peak voltage between 1.0 and $1.3 \mathrm{~V}$ was applied between source and drain contacts, while $\mathrm{a} \approx 50-\mu \mathrm{l}$ drop of CNT dispersion with a concentration of $\approx 5$ CNTs per $\mu \mathrm{m}^{3}$ was placed on the device. After $5 \mathrm{~min}$, the drop was first diluted with doubly distilled water, followed by methanol and finally allowed to dry.

Before the samples were transferred to the HIM, CNT deposition was assessed with a conventional scanning electron microscope (SEM), see Fig. 1(b). To eliminate any effects from electron-beam exposure, samples were annealed in a vacuum oven ( $p=10^{-6}$ millibars) at $600^{\circ} \mathrm{C}$ for $30 \mathrm{~min}$ after imaging. Electrical characterization of pristine devices showed an Ohmic current-voltage behavior with a resistance of, typically, $500 \mathrm{k} \Omega$.

The Zeiss Orion Plus HIM used in this work allows imaging similar to an SEM, except for a helium ion beam being scanned over the sample. The image is generated by the detection of secondary electrons from the sample. Due to the very small effective source size, favorable beam-sample interaction and a much smaller de Broglie wavelength of helium ions compared to electrons, the HIM offers an improved resolution compared to traditional SEMs. ${ }^{8,9}$ The acceleration voltage of the HIM was always set to $35 \mathrm{kV}$. The $5-\mu \mathrm{m}$ aperture was used, resulting in a spot size of below $1 \mathrm{~nm}$ and a beam current of $\approx 0.4 \mathrm{pA}$. The signal-to-noise ratio of an image and the implanted ion dose is depend on the beam current, the dwell time per pixel and the averaging settings. To minimize the ion dose, fast alignment images were recorded using the following settings: Pixel spacing $1 \mathrm{~nm}$, dwell time $0.5 \mu \mathrm{s}$, no averaging. This led to a line dose of $0.2 \mathrm{nC} / \mathrm{m}$ per scan line. Later, slow scans for characterization were performed with fixed parameters: A pixel spacing of $5 \AA$, a dwell time of $0.5 \mu \mathrm{s}$, and $32 \times$ line averaging. This led to a line dose of $13 \mathrm{nC} / \mathrm{m}$ per scan line.
The focused helium ion beam can also be used to pattern samples by physical sputtering, similar to gallium ions in a focused ion beam instrument. This has been demonstrated, e.g., for graphene, ${ }^{10-12}$ silicon nitride, ${ }^{13}$ and recently gold nanorods. ${ }^{14}$ Here, we employed helium ion beam lithography to pattern nanogaps into metallic carbon nanotubes, in a device geometry. These nanogaps were then used as contacts for a molecular wire, to demonstrate their practical usage. To reduce hydrocarbon deposition on the surface, all samples were stored in the helium ion microscope chamber under high vacuum for at least several hours. The chamber pressure, typically, reached $2.5 \times 10^{-7}$ millibars before experiments were started.

In order to cut CNTs, a single pixel line with a pixel spacing of $2.5 \AA$ and a dwell time in the millisecond range was scanned across a nanotube. To align this line perpendicularly to the nanotube, a fast scan was performed before the lithography was started, see Fig. 1(c).

To ascertain the critical dose for gap formation, we employed voltage-contrast microscopy (VCSEM), which is capable of locating defects and gaps within a nanotube and to reveal the nanotube's electronic type. ${ }^{15}$ In our devices, this was realized by grounding one of the two metal electrodes. The other electrode remained floating, albeit connected to the grounded electrode by the mSWNT. After a single pixel line was scanned across the nanotube, a slow scan image of the device was acquired. Once an electrically insulating nanogap was formed in the metallic CNT, the floating electrode accumulated positive charges, thereby inhibiting secondary electrons from reaching the detector. Thus, the floating electrode appeared darker in the image, while the grounded electrode and the CNT segment connected to it appeared brighter, see Fig. 1(d) for an example. Using this experimental procedure, the critical dose for gap formation was determined to be $\approx 24 \mu \mathrm{C} / \mathrm{m}$. Before proceeding to cut further nanogaps, the beam current was measured and the pixel dwell time adjusted accordingly to accommodate this value. We note that the line doses implanted by our fast- and slow-scan images are at least three orders of magnitude lower, and thus, have a negligible sputtering effect.

In order to precisely measure the size of the gap formed, the electrostatic charging of floating electrodes had to be 
avoided as charging always caused drifts. Also, the different secondary electron intensities on opposite sides of the gap would make an analysis difficult. To avoid charging, the samples were mounted in commercial 16-pin dual in-line package chip carriers, where the common drain electrode and source electrodes of devices were bonded to the chip carrier with $\mathrm{Al}$ wires. A customized sample holder in the HIM connected all pins to the stage/ground potential. In this way, detailed slow scans of the nanogaps after lithography were made possible, see Fig. 1(e).

Secondary electron intensity profiles were then recorded across 14 nanogaps in different carbon nanotube devices. The resulting curves were fitted with inverted Gaussians, see Fig. 2(a). A histogram of FWHM of the nanogaps is plotted in Fig. 2(b). With an average nanogap size of $(2.8 \pm 0.6)$ $\mathrm{nm}$, direct helium ion sputtering is more precise by almost an order of magnitude than electron-beam-induced etching and a factor of 2-3 better than the smallest gaps achievable by current-induced breakdown. Most striking is the comparably narrow gap size distribution, which is an indication of the highly reproducible nature of this method. As we were targeting the smallest gap size possible for the subsequent insertion of molecules, we did not explore the formation of larger gaps.

Electrical measurements were performed on pristine mSWNTs and on mSWNTs after gap formation. The resistance of pristine mSWNTs yielded $(479 \pm 193) \mathrm{k} \Omega$ and is comparable to $1.2 \mathrm{~nm}$ diameter mSWNTs on Pd electrodes. ${ }^{16}$ mSWNTs with nanogaps had a resistance of $(643 \pm 311) \mathrm{T} \Omega$, which is nine orders of magnitude higher than in pristine
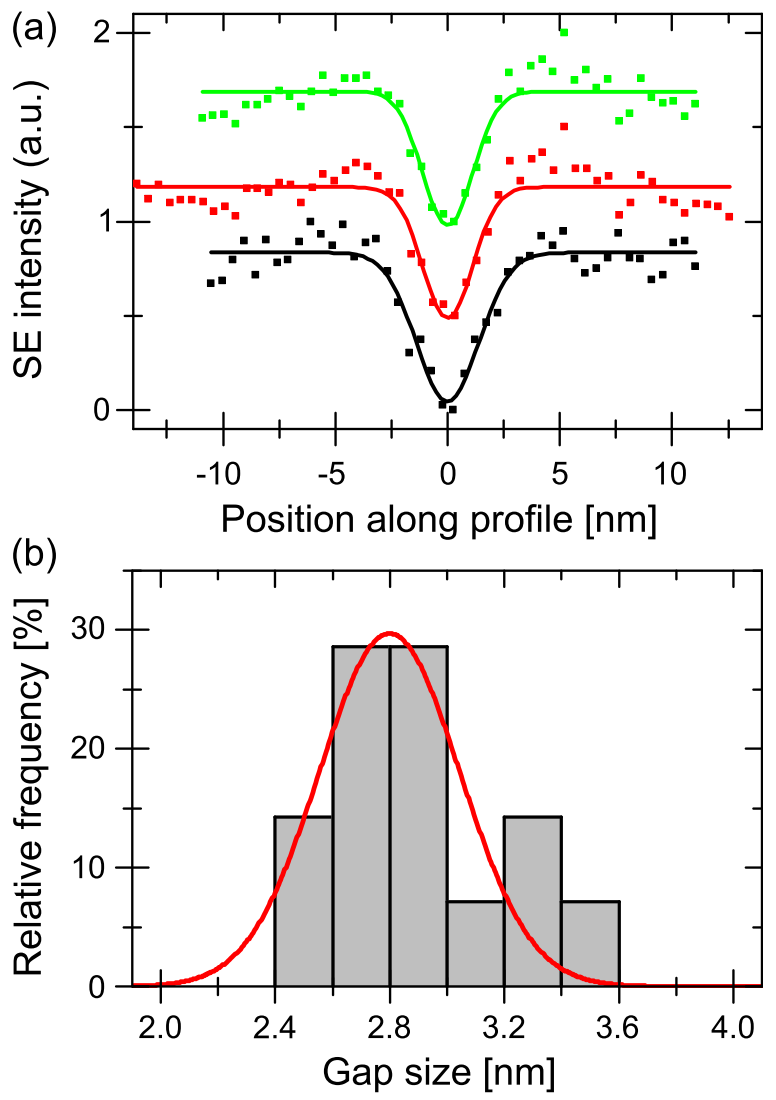

FIG. 2. (a) Secondary-electron intensity profiles from three different nanogaps. (b) Histogram of 14 nanogap sizes.

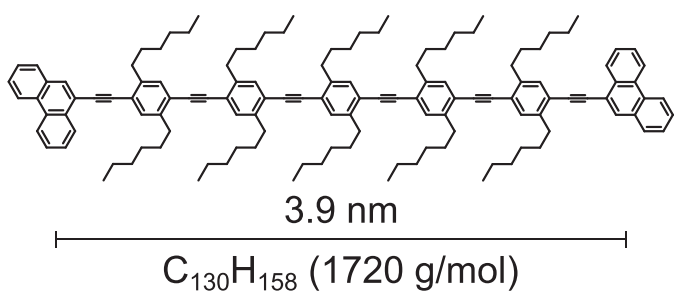

FIG. 3. Structure of OPE Rod 1 which was contacted by the nanogaps. Phenanthrene anchor groups on each side couple to the sidewalls of a carbon nanotube. ${ }^{17}$

devices. We attempted to measure tunneling or field-emission currents through the air gap and to correlate the current with the HIM derived gap size. However, despite the large electric fields of up to $5 \mathrm{~V} / \mathrm{nm}$ in these nanogaps, we were not able to detect any sign of field emission.

To demonstrate the utility of these nanogaps for contacting organic molecules, OPE Rod 1, a symmetric molecular wire of $3.9 \mathrm{~nm}$ length, with five subunits and phenanthrene anchor groups at each end was synthesized by performing a series of acetylene protection and deprotection steps, ${ }^{18}$ similar to the molecule used by Grunder et al., ${ }^{19}$ see Fig. 3. A voltage of $1 \mathrm{~V}$ was applied across the nanogap device and the current monitored, and a drop of very dilute OPE Rod 1 solution (less than $1 \mu \mathrm{g} / \mathrm{ml}$ in methylene chloride) was placed on the device and allowed to dry under ambient conditions. This process took, typically, no longer than $2 \mathrm{~min}$, after which the devices exhibited a low-bias resistance of $(90 \pm 85) \mathrm{G} \Omega$. See Fig. 4 for a resistance histogram over the lifetime of nanogap devices, including 50 devices with OPE molecules. For reference, experiments with clean solvent without the OPE molecule, the conductance of a nanogap was not changed. Interestingly, it was possible to wash off contacted molecules using clean solvent, thereby, restoring the conductance of a device to the level of an empty nanogap.

Scanning tunneling microscope break-junction measurements on an OPE of similar length revealed a resistance of $\approx 200 \mathrm{M} \Omega$ for a single molecule, albeit with the molecule covalently bonded to gold on both sides. ${ }^{20}$ We observe an

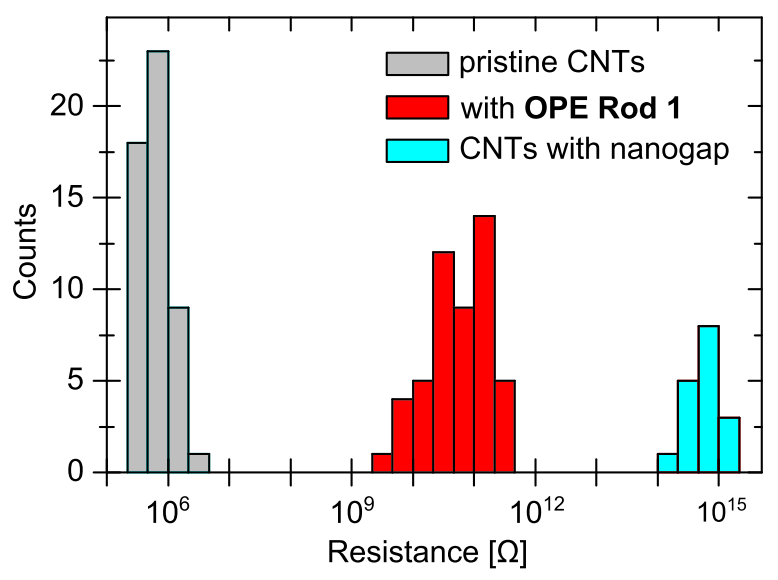

FIG. 4. Resistance histogram over the lifetime of nanogap electrode devices, including 50 CNT-OPE-CNT contacts. Pristine mSWNT devices: $(479 \pm 193) \mathrm{k} \Omega$; CNTs with nanogap: $(643 \pm 311) \mathrm{T} \Omega$; with OPE molecules: $(90 \pm 85) \mathrm{G} \Omega$. 
average resistance almost three orders of magnitude higher, with values spread over roughly two orders of magnitude. We ascribe this to a varying imperfect attachment of the molecules' anchor groups to the CNT electrodes and conformational freedom of molecules in the junction; both profoundly deteriorating the conductance. ${ }^{21}$ Currently, STM investigations of the molecule and its electronic properties are underway to resolve these issues.

The reproducible engineering of nanogaps in carbon nanotubes that we have achieved will allow the study of many other organic or inorganic systems of nanoscale dimensions at the single-molecule or few-atom level, by providing a reliable way to fabricate nanoscale electrodes. Future work will have to address the issue of establishing reliable contacts between CNT electrodes and molecules.

${ }^{1}$ P. Qi, A. Javey, M. Rolandi, Q. Wang, E. Yenilmez, and H. Dai, J. Am. Chem. Soc. 126, 11774 (2004).

${ }^{2}$ C. W. Marquardt, S. Grunder, A. Błaszczyk, S. Dehm, F. Hennrich, H. v. Löhneysen, M. Mayor, and R. Krupke, Nat. Nanotechnol. 5, 863-867 (2010). ${ }^{3}$ F. Xiong, A. D. Liao, D. Estrada, and E. Pop, Science 332, 568-570 (2011).

${ }^{4}$ C. Thiele, M. Engel, F. Hennrich, M. M. Kappes, K.-P. Johnsen, C. G. Frase, H. v. Löhneysen, and R. Krupke, Appl. Phys. Lett. 99, 173105 (2011).

${ }^{5}$ S. Lebedkin, P. Schweiss, B. Renker, S. Malik, F. Hennrich, M. Neumaier, C. Stoermer, and M. M. Kappes, Carbon 40, 417-423 (2002).

${ }^{6}$ K. Moshammer, F. Hennrich, and M. M. Kappes, Nano Res. 2, 599-606 (2009).
${ }^{7}$ A. Vijayaraghavan, S. Blatt, D. Weissenberger, M. Oron-Carl, F. Hennrich, D. Gerthsen, H. Hahn, and R. Krupke, Nano Lett. 7, 1556 (2007).

${ }^{8}$ D. C. Bell, Microsc. Microanal. 15, 147 (2009).

${ }^{9}$ R. Hill, J. A. Notte, and L. Scipioni, in Advances in Imaging and Electron Physics, edited by P. W. Hawkes (Elsevier, 2012), Vol. 170, pp. 65-148.

${ }^{10}$ D. Bell, M. Lemme, L. Stern, J. Williams, and C. Marcus, Nanotechnology 20, 455301 (2009).

${ }^{11}$ M. Lemme, D. Bell, J. Williams, L. Stern, B. Baugher, P. Jarillo-Herrero, and C. Marcus, ACS Nano 3, 2674 (2009).

${ }^{12}$ D. Fox, Y. B. Zhou, A. O'Neill, S. Kumar, J. J. Wang, J. N. Coleman, G. S. Duesberg, J. F. Donegan, and H. Z. Zhang, Nanotechnology 24, 335702 (2013).

${ }^{13}$ M. M. Marshall, J. Yang, and A. R. Hall, Scanning 34, 101 (2012).

${ }^{14}$ O. Scholder, K. Jefimovs, I. Shorubalko, C. Hafner, U. Sennhauser, and G.-L. Bona, Nanotechnology 24, 395301 (2013).

${ }^{15}$ A. Vijayaraghavan, S. Blatt, C. Marquardt, S. Dehm, R. Wahi, F. Hennrich, and R. Krupke, Nano Res. 1, 321 (2008).

${ }^{16}$ W. Kim, A. Javey, R. Tu, J. Cao, Q. Wang, and H. Dai, Appl. Phys. Lett. 87, 173101 (2005).

${ }^{17}$ S. Gotovac, Y. Hattori, D. Noguchi, J.-I. Miyamoto, M. Kanamaru, S. Utsumi, H. Kanoh, and K. Kaneko, J. Phys. Chem. B 110, 16219 (2006).

${ }^{18}$ See supplementary material at http://dx.doi.org/10.1063/1.4868097 for full synthetic details.

${ }^{19}$ S. Grunder, D. Muñoz Torres, C. Marquardt, A. Blaszczyk, R. Krupke, and M. Mayor, Eur. J. Org. Chem. 2011, 478 (2011).

${ }^{20}$ Q. Lu, K. Liu, H. Zhang, Z. Du, X. Wang, and F. Wang, ACS Nano 3, 3861 (2009).

${ }^{21}$ L. Venkataraman, J. E. Klare, C. Nuckolls, M. S. Hybertsen, and M. L. Steigerwald, Nature 442, 904 (2006). 